\title{
ИССЛЕДОВАНИЕ ВОЗМОЖНОСТИ ИСПОЛЬЗОВАНИЯ РЕКУРРЕНТНОЙ НЕЙРОННОЙ СЕТИ ХОПФИЛДА ДЛЯ РЕШЕНИЯ ЗАДАЧИ КОММИВОЯЖЕРА
}

\section{USING POSSIBILITY INVESTIGATION OF RECURRENT HOPFIELD NETWORK TO TRAVELLING SALESMAN PROBLEM SOLVING}

\section{Muzychin S. Matsievsky}

Summary. The first part such well-known concepts as Hopfield neural network, dynamical system, travelling salesman problem are introduced and explained.

The main part a specific Hopfield network is built, the setting of the travelling salesman problem is given and its mathematical model on the basis of the Hopfield neural network is built. Numerical methods are proposed to solve the proposed mathematical model.

Keywords: Hopfield network, dynamical system, travelling salesman problem, mathematical model, numerical analysis.

\author{
Музычин Владимир Витальевич \\ Аспирант, Балтийский федеральный университет \\ им. И. Канта (Калининград) \\ mv@1c39.ru \\ Мациевский Сергей Валентинович \\ К.ф.-м.н., дочент, Балтийский федеральный \\ университет им. И. Канта (Калининград) \\ sergei.matsievsky@ya.ru
}

Аннотация. В первой части вводятся и поясняются такие известные понятия, как нейронная сеть Хопфилда, динамическая система, задача коммивояжера.

В содержательной части строится конкретная сеть Хопфилда, дается постановка задачи коммивояжера и строится ее математическая модель на основе нейросети Хопфилда. Для решения предложенной математической модели предлагаются численные методы.

Ключевые слова: нейронная сеть Хопфилда, динамическая система, задача коммивояжера, математическая модель, численный метод.

\section{1. Нейронная сеть Хопфи^Аа}

В 1982 г. (в год полного парада планет) Джон Джозеф Хопфилд [2]:

1. вышел на новый уровень понимания вычислений, которые происходят при работе рекуррентных нейронных сетей, обладающими симметричными синаптическими связями, применив функцию энергии;

2. доказал изоморфизм между моделью Изинга (Ising model) статистической физики и рекуррентной нейронной сетью.

Этот изоморфизм привлек физиков и их результаты в область искусственных нейросетей. Таким образом, в 1980-х годах нейросети с обратной связью получили много внимания и стали называться нейронными сетями Хопфилда (Hopfield network). Сети Хопфилда не являются реалистичными моделями нейробиологических систем, однако они, как динамически устойчивые системы, могут хранить информацию [1, с. 79].

Искусственная нейронная сеть Хопфилда однослойна и имеет систему единичных задержек, определяющую 


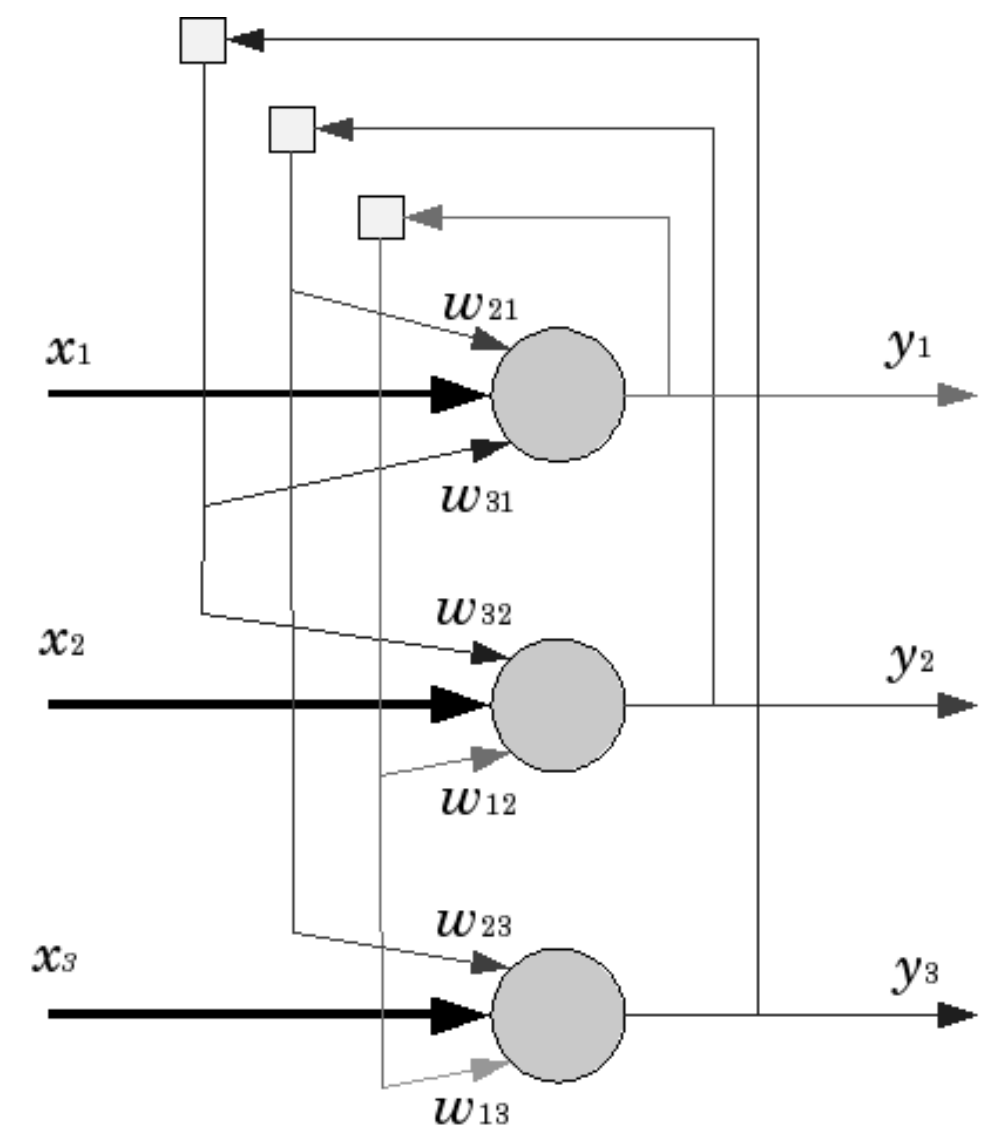

Рис. 1. Схема сети Хопфилда с тремя нейронами. Взято со Склада Википедии: URL: https://commons.wikimedia.org/wiki/File: Hopfield\%27s_net.png

множественные обратные связи (multiple-loop feedback system) (рис. 1). Обратных связей столько, сколько нейронов в слое: выход каждого нейрона замыкается на все остальные с единичной задержкой (нейрон не имеет обратной связи с самим собой) [1, с. 856].

Итак, основное применение дискретной сети Хопфилда состоит в моделировании контентно-адресуемой (ассоциативной, адресноориентированной) (contentaddressable memory) памяти [1, с. 862], основанной на нейроне Мак-Каллока - Питца [3], т.е. на нейроне с жестко ограниченной функцией активации [1, с. 909].

Рассматривая эту модель Хопфилда в вычислительном контексте, получаем довольно тривиальную конструкцию. Тем не менее эта модель ассоциативной памяти достаточна важна, поскольку объясняет связь между динамикой и вычислениями по-новому. Например, она дает понимание следующих трех свойств, которые имеют нейробиологическую релевантность:

1. динамика модели Хопфилда регулируется множеством точечных аттракторов - ячеек фундаментальной памяти - в многомерном пространстве состояний;
2. нужный точечный аттрактор находится простой инициализации модели приблизительным описанием, затем динамика модели автоматически переведет состояние модели к ближайшему точечному аттрактору;

3. обучение - вычисление свободных параметров модели - определяется постулатом обучения Хебба, причем с помощью этого механизма можно добавлять новые точки аттракторов [1, с. 909].

\section{2. Аинамическал система}

Для изучения нейродинамики требуется математическая модель, которая описывает динамику нелинейной системы. Для этого вполне подходит модель в пространстве состояний (state-space model), а рассуждения проводятся в терминах переменных состояний (state variable), значения которых в любой момент времени содержат данные, достаточные для предсказания эволюции системы [1, с. 837].

Динамика многих нелинейных динамических систем описывается системой дифференциальных уравнений первого порядка: 


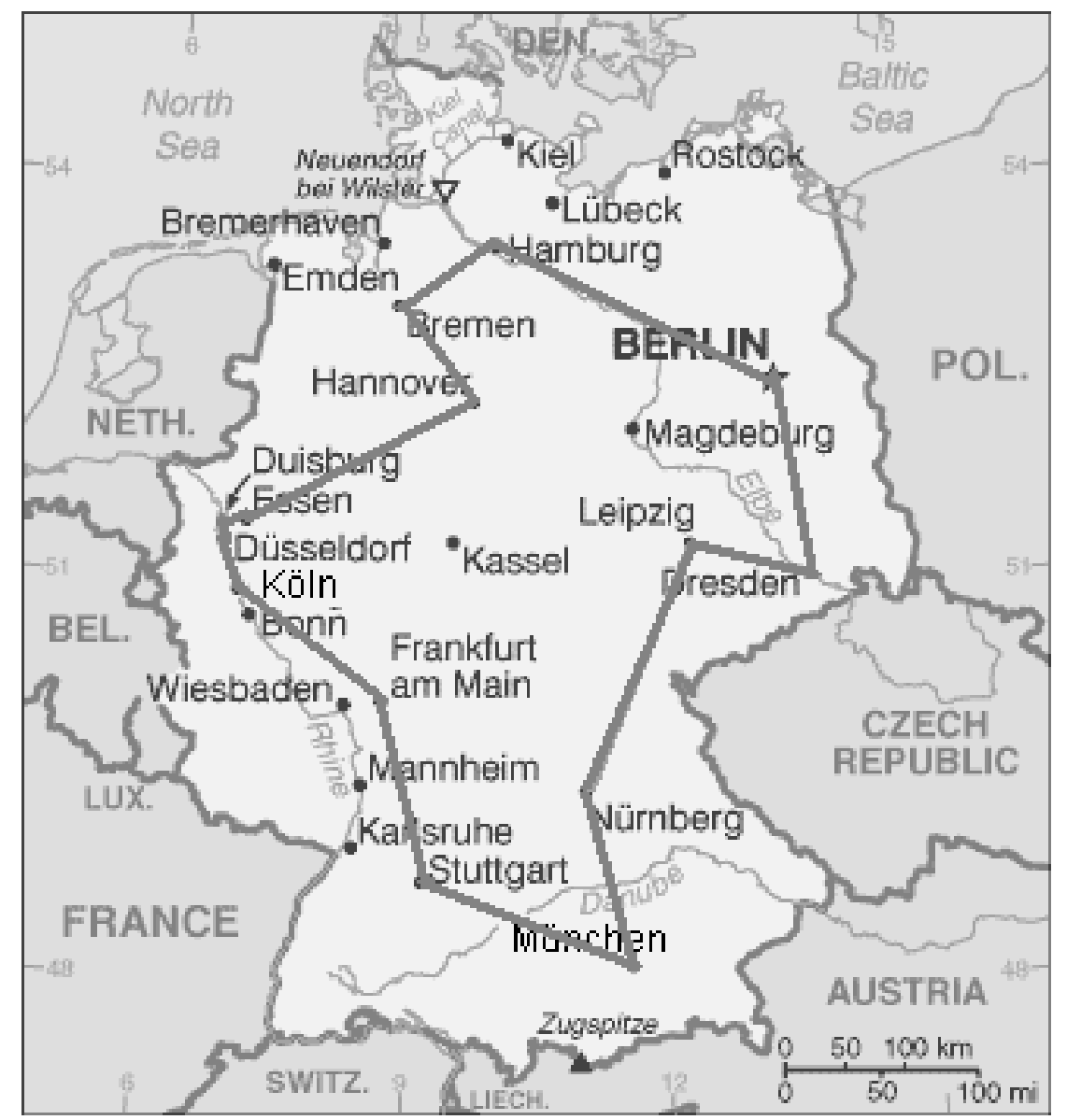

Рис. 2. Оптимальный маршрут коммивояжера через 15 крупнейших городов Германии - самый короткий из всех возможных 43589145 600. Взято со Склада Википедии: URL: https://commons.wikimedia.org/wiki/File:TSP_Deutschland_3.png

$\frac{d}{d t} x_{j}(t)=F_{j}\left(x_{j}(t)\right), j=1,2, \ldots, N$,

где $F_{j}(\cdot)$ - некоторая нелинейная функция; $x_{j}(t)-$ переменные состояния динамической системы; $t$ - независимая переменная непрерывного времени; $N$ - порядок системы.

Для удобства выкладок переменные состояния можно собрать в вектор $x(t)$ размерности $N \times 1$ в вектор состояний (state vector) динамической системы. Тогда в векторных обозначениях система дифференциальных уравнений примет более компактный вид

$$
\frac{d}{d t} \mathbf{x}(t)=\mathrm{F}(\mathbf{x}(t))
$$

где F —нелинейная вектор-функция, каждый элемент которой связан с соответствующим элементом вектора состояний

$$
\mathbf{x}(t)=\left[x_{1}(t), x_{2}(t), \ldots, x_{N}(t)\right]^{T} .
$$

Определение автономной системы. Нелинейная динамическая система, в которой вектор-функция $\mathrm{F}(\mathrm{x}(\mathrm{t}))$ не зависит явным образом от времени $t$, называется автономной (autonomic). В противном случае система называется неавтономной.

Неавтономная динамическая система имеет уравнение состояния

$$
\frac{d}{d t} \mathbf{x}(t)=\mathrm{F}(\mathbf{x}(t), t)
$$

с начальным состоянием $\mathbf{x}\left(t_{0}\right)=\mathbf{x}_{0}$. В неавтономных системах вектор поля $\mathrm{F}(\mathrm{x}(\mathrm{t}), \mathrm{t})$ зависит от времени $\mathrm{t}$. Таким образом, в отличие от автономных систем в общем случае нельзя принять начальное время равным нулю [4].

Далее рассматриваются только автономные системы.

При любой функции $\mathrm{F}(\mathrm{x}(\mathrm{t}))$ вектор состояния $\mathrm{x}(\mathrm{t})$ может зависеть от времени t. В противном случае состоя- 
ние $x(t)$ есть константа и система не является динамической.

Определение динамической системы. Динамической система - это система, состояния которой изменяются со временем

Более того,

$$
\frac{d}{d t} \mathbf{x}(t)
$$

можно представить как вектор скорости не в физическом, а в абстрактном смысле. Тогда, исходя из

$$
\frac{d}{d t} \mathbf{x}(t)=\mathrm{F}(\mathbf{x}(t), t)
$$

вектор-функция $\mathrm{F}(\mathrm{x}(\mathrm{t}))$ называется векторным полем скорости (velocity vector field), или даже просто полем скорости.

\section{3. Захача коммивояжера}

Еще одно применение сетей Хопфилда - решение некоторых задач комбинаторной оптимизации (combinatorial optimization problems), включающих классическую задачу коммивояжера (Travelling Salesman Problem, TSP). На плоскости расположено множество городов, требуется найти кратчайший маршрут обхода всех городов, при этом вернувшись в первый город (рис. 2).

При сравнительно простой формулировке само решение TSP является крайне сложным, это задача считается NP- полной (NP-complete). В исследовании [5] было показано, как с помощью аналоговой сети, основанной на дифференциальных уравнениях 1-го порядка, представить решение TSP. В частности, синаптические веса получаются из расстояний между городами, а оптимальное решение - это фиксированная точка нейродинамических уравнений [1, с. 909].

Именно здесь и собраны сложности, связанные с переформулировкой комбинаторных задач оптимизации в терминах непрерывных (аналоговых) сетей Хопфилда. Сеть Хопфилда минимизирует только одну функцию энергии (по Ляпунову), тогда как стандартная комбинаторная задача оптимизации оптимизирует целевую функцию при наличии множества целевых ограничений [6]. Причем при нарушении любого ограничения решение считается неверным.

Первые опыты такой переформулировки использовали функцию Ляпунова, построенной специальным методом при одном слагаемом для каждого условия:

$$
E=E^{\text {опт. }}+A E_{A}^{\text {oгр. }}+B E_{B}^{\text {огр. }}+C E_{C}^{\text {огр. }}+\ldots
$$

Первое слагаемое $E^{\text {onm. }}$ есть минимизируемая целевая функция (например, длина маршрута в TSP) и определяется поставленной задачей. Оставшиеся слагаемые $E_{A}^{o r p .}, E_{B}^{o r p .}, E_{C}^{o r p .}$ суть штрафные функции, минимизация которых удовлетворяет ограничениям. Скаляры $A, B, C$ суть постоянные веса, назначенные соответствующим штрафным функциям и определяемые методом проб и ошибок. К сожалению, многочисленные слагаемые в вышеприведенной функции Ляпунова вытесняют друг друга, поэтому успех сети Хопфилда значительно зависит от относительных значений A, B, C [6].

\section{4. Построение сети Хопфильа}

Решение любой задачи с помощью сети Хопфилда сводится поиску минимума функционала, называемого энергией сети. В рассматриваемом случае сеть Хопфилда сходится к локально-оптимальному решению задачи. При этом промежуточные состояния нейронов образуют не само решение, а приближение к нему, которое задается матрицей действительных чисел из отрезка $[0,1]$.

Для каждого нейрона в сети так называемый входной и выходные потенциаль могут быть определены и обозначены $x$ и $y$ соответственно (см. рис. 1).

В сети Хопфилда функция активации обычно S-образная. В этой статье положим функцию активации одинаковую для всех нейронов и равную

$$
y(x)=\frac{1}{2}(1+\operatorname{th}(\alpha x)),
$$

где $\alpha$ - некоторый параметр этой функции. При $\alpha=1$ график изображен на рисунке 3.

Для достаточно больших значений $\alpha$ приближенные значения $y$ описываются формулой

$$
y(x)=\left\{\begin{array}{l}
0, \text { если } x<0, \\
1, \text { если } x>0 .
\end{array}\right.
$$

Далее, если однослойная сеть Хопфилда состоит из $n$ нейронов, то функция энергии $E$ сети находится по формуле

$$
E=-\frac{1}{2} \sum_{i=1}^{n} \sum_{j=1}^{n} w_{i j} y_{i} y_{j},
$$

где $w_{i j}, i, j=1, \ldots, n-$ вес соединения между выходом $j$-го нейрона $y_{i j}$ и входом $i$-го нейрона $x_{i j}$.

Все веса $w_{i j}$ образуют матрицу весов соединений.

Эти веса могут быть:

1) положительными - стимулирующий стимул;

2) отрицательными - тормозной стимул; 


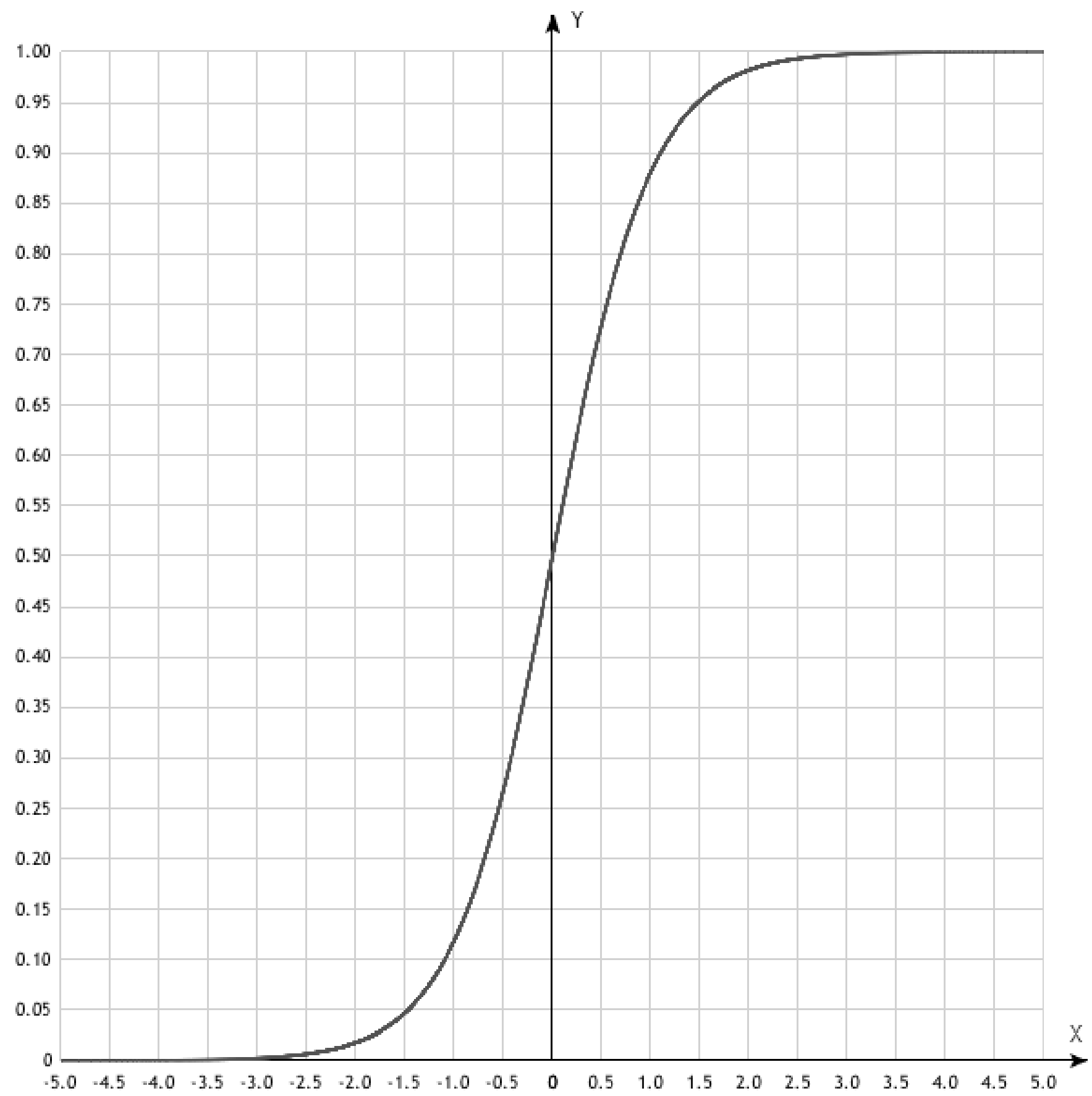

Рис. 3. График функции активации нейронов $y(x)=\frac{1}{2}(1+\operatorname{th}(\alpha x))$ при параметре $\alpha=1$

3) равными нулю - отсутствие связи от нейрона $j$ к нейрону $i$.

Входной потенциал $i$-го нейрона определяется уравнением

$x_{i}=\frac{\partial E}{\partial y_{i}}, i=1, \ldots, n$.

Из последних двух уравнений получаем:

$$
x_{i}=-\sum_{j=1}^{n} w_{i j} y_{i}, i=1, \ldots, n \text {. }
$$

5. Постановка TSP

Вышеуказанные правила использовались различными авторами в попытках решить сложную проблему комбинаторной оптимизации. Наибольшее внимание среди 
них, вероятно, было уделено задаче коммивояжера, которая может быть сформулирована так.

Определение TSP. Для заданного набора из $n$ городов и заданных расстояний между каждыми двумя из них необходимо найти замкнутый маршрут минимальной длины, проходящий через каждый город только один раз.

На основе терминологии теории графов перепишем это определение на графе.

Определение TSP на графе. Для заданного полного графа $K_{n}$ и симметричной матрицы, представляющей веса его ребер, необходимо найти гамильтонов цикл в $K_{n}$ минимальной длины (стоимости).

Примечание 1. Представленное здесь определение TSP - не единственная возможная версия. В других постановках матрица не является симметричной или не все города связаны ребром. Некоторые альтернативные определения, а также основные общеизвестные теоретические результаты, касающиеся вычислительной сложности различных версий TSP, можно найти в статье [7].

Примечание 2. Так как два вышеупомянутых определения эквивалентны, будем использовать терминологию из определения 1.

Из-за предполагаемой неполиномиальной сложности TSP традиционные подходы для решения проблемы основаны либо на методах перебора, либо на эвристике. В этой работе идея эволюции сети, а также способ выбора отправной точки моделирования идентичны тем, которые использовались для задачи расстановки N королев (NQP) в статье [8].

Далее, в данной статье любое решение цикла TSP (гамильтонов граф по всем городам) будет рассматриваться как решение проблемы. Решения, которые минимизируют длину маршрута, назовем лучиими решениями. Очевидно, что для любого решения существуют $2 n-1$ других решений такой же длины, которые отличаются друг от друга начальным городом или направлением движения. Ради простоты все они будут рассматриваться как одно и то же решение.

\section{6. Математическая модель}

Решение проблем оптимизации с сетью Хопфилда требует осторожного и адекватного выбора энергетической функции $E$, то есть весов $w_{i j}$. Функция $E$ должна быть определена таким образом, чтобы ее минимумы соответствовали решениям рассматриваемой задачи.

\section{В этой статье $E$ имеет вид}

$$
E=E_{1}+E_{2}
$$

где

$$
\begin{aligned}
& E_{1}=\frac{A}{2} \sum_{i=1}^{n} \sum_{j=1}^{n} \sum_{\substack{k=1 \\
k \neq j}}^{n} y_{i j} y_{i k}+\frac{B}{2} \sum_{i=1}^{n} \sum_{j=1}^{n} \sum_{\substack{k=1 \\
k \neq j}}^{n} y_{j i} y_{k i}+ \\
& +\frac{C}{2}\left(\sum_{i=1}^{n} \sum_{j=1}^{n} y_{i j}-(n+\sigma)\right)^{2}, \\
& E_{2}=\frac{D}{2} \sum_{i=1}^{n} \sum_{\substack{j=1 \\
j \neq i}}^{n} \sum_{k=1}^{n} d_{i j} y_{i k}\left(y_{j, k+1}+y_{j, k-1}\right),
\end{aligned}
$$

$n$ - число городов; $d_{i j}$ - расстояние между городами $i$ и $j ; y_{i j}$ - матрица номеров городов в циклическом маршруте обхода: $y_{i j}=1$ означает, что город $i$ будет $j$-м по счету, все остальные $y_{i j}$ равны нулю [5].

Способ решения TSP, представленный здесь, отличается от классического подхода механизмом изменения выхода нейронов и выражается формулой

$$
\begin{aligned}
& \frac{d x_{i j}}{d t}=-\frac{x_{i j}}{\tau}-A \sum_{\substack{k=1 \\
k \neq j}}^{n} y_{i k}-B \sum_{\substack{k=1 \\
k \neq i}}^{n} y_{k j}- \\
& -C\left(\sum_{i=1}^{n} \sum_{j=1}^{n} y_{i j}-(n+\sigma)\right)- \\
& -D \sum_{k=1}^{n} d_{i k}\left(y_{k, j+1}+y_{k, j-1}\right) .
\end{aligned}
$$

\section{7. Численные метолы}

Для практической компьютерной реализации представим последнее уравнение в разностной форме:

$$
\begin{aligned}
& x_{i j}(t+\Delta t)=x_{i j}(t)+\Delta t \times \\
& \times\left(-x_{i j}(t)-A \sum_{\substack{k=1 \\
k \neq j}}^{n} y_{i k}(t)-B \sum_{\substack{k=1 \\
k \neq i}}^{n} y_{k j}(t)-\right. \\
& -C\left(\sum_{i=1}^{n} \sum_{j=1}^{n} y_{i j}(t)-(n+\sigma)\right)- \\
& \left.-D \sum_{k=1}^{n} d_{i k}\left(y_{k, j+1}(t)+y_{k, j-1}(t)\right)\right) .
\end{aligned}
$$

В этом уравнении состояние нейрона $i j, i, j=1, \ldots, n$, в момент времени $t+1$ зависит от его состояния в момент времени $t$. В наших моделях входной потенциал в момент времени $t+1$ не напрямую зависит от его состояния в предыдущий момент. На самом деле 


$$
\begin{aligned}
& x_{i j}(t+1)=-A \sum_{\substack{k=1 \\
k \neq j}}^{n} y_{i k}(t)-B \sum_{\substack{k=1 \\
k \neq i}}^{n} y_{k j}(t)- \\
& -C\left(\sum_{i=1}^{n} \sum_{j=1}^{n} y_{i j}(t)-(n+\sigma)\right)- \\
& -D \sum_{k=1}^{n} d_{i k}\left(y_{k, j+1}(t)+y_{k, j-1}(t)\right) .
\end{aligned}
$$

Представленное выше правило обновления также использовалось в статье [9]. Авторы сообщают о 50\% сходимости к гамильтоновому циклу. Другими словами, в половине случаев решение не было найдено. Лучшая сходимость, полученная в наших моделях, обусловлена лучшим выбором сетевых констант.

Самым большим преимуществом предлагаемой сети является ее независимость от начального состояния, т.е. выходных потенциалов нейронов в начале моделирования.

Результаты компьютерного моделирования, а также обсуждение влияния сетевых констант будут представлены отдельно.

\section{ЛИТЕРАТУРА}

1. Хайкин С. Нейронные сети: полный курс. М.: 000 «И. Д. Вильямс», 2006. (Haykin S. Neural Networks. A Comprehensive Foundation. Prentice Hall, NJ: Upper Saddle River, 1998.)

2. Hopfield J. J. Neural networks and physical systems with emergent collective computational abilities // Proceedings of the National Academy of Sciences, USA. 1982. Vol. 79. P. 2554-2558. doi:10.1073/pnas.79.8.2554. URL: https://bi.snu.ac.kr/Courses/g-ai09-2/hopfield82.pdf

3. Мак-Каллок У.С., Питтс В. Логическое исчисление идей, относящихся к нервной активности // Автоматы / Пол ред. К. э. Шеннона и Дж. Маккарти. М.: Издво ИЛ, 1956. С. 363-384. (Также в журнале Нейрокомпьютер, 1992, № 3/4, С. 40-53, и в книге Нейронные сети: история развития теории. М.: ИПРЖР, 2001. C. 5-22.) (McCulloch W. S., Pitts W.A logical calculus of the ideas immanent in nervous activity // Bulletin of Mathematical Biophysics. 1943. Vol. 5. P. 115-133.)

4. Parker T. S., Chua L. 0. Practical Numerical Algorithms for Chaotic Systems. New York: Springer, 1989.

5. Hopfield J. J., Tank T.W. “Neural" computation of decisions in optimization problems // Biological Cybernetics. 1985. Vol. 52(3). P. 141-152. URL: https://link.springer. com/article/10.1007/BF00339943

6. Gee A. H., Aiyer S. V.B., Prager R. An analytical framework for optimizing neural networks // Neural Networks. 1993. Vol. 6. P. 79-97.

7. Reingold E. M., Nievergelt J., Deo N. Algorytmy kombinatoryczne, PWN. Warszawa, 1985.

8. Mandziuk J. Solving the N-Queens problem with a binary Hopfield-ty pe network. Synchronous and asynchronous model // Biological Cybernetics. 1995. Vol. 72, No. 5. P. 439-446.

9. Yao K. C., Chavel P., Meyrueis P. Perspective of a neural optical solution to the Traveling Salesman optimization Problem // SPIE. 1989. Vol. 1134. Optical Pattern Recognition II. P. 17-25.

(с) Музычин Владимир Витальевич ( mv@1с39.ru ), Мациевский Сергей Валентинович ( sergei.matsievsky@ya.ru ).

Журнал «Современная наука: актуальные проблемы теории и практики» 